\title{
La terapia cognitiva basada en la conciencia plena no fue superior a los antidepresivos en la prevención de la depresión recurrente
}

Mindfulness based cognitive therapy was not superior to antidepressants for the prevention of recurrent depression

Kuyken W y col. The Lancet 2015;386(9988):63-73.

\section{Objetivos}

Comparar la efectividad de la terapia cognitiva basada en la conciencia plena (TCBCP) asociada a la reducción paulatina y discontinuación de la medicación antidepresiva, contra el mantenimiento de la medicación antidepresiva, para prevenir la recurrencia de la depresión en pacientes con historia de tres o más episodios previos.

\section{Diseño y lugar}

Ensayo clínico aleatorizado simple ciego, realizado en cuatro centros de atención primaria (con población rural y urbana) del Reino Unido.

\section{Pacientes}

Se incluyeron 424 pacientes, mayores de 18 años, con diagnóstico de trastorno depresivo mayor recurrente (tres o más episodios) en remisión total o parcial según criterios del Manual Diagnóstico y Estadístico de los Trastornos mentales (sigla en inglés, DSM-IV), que estuviesen recibiendo medicación antidepresiva. Se excluyeron pacientes con un episodio depresivo mayor en curso, abuso de sustancias, daño cerebral estructural, psicosis, enfermedad bipolar, comportamiento antisocial persistente, comportamiento auto-agresivo o psicoterapia formal vigente.

\section{Intervención}

Los pacientes fueron aleatorizados a recibir TCBCP sumado a un asesoramiento para reducir paulatinamente y discontinuar su medicación antidepresiva (grupo intervención) o a continuar con su medicación antidepresiva (grupo control), durante dos años. El programa de TCBCP consistió en ocho sesiones semanales de 145 minutos de duración, seguidas de cuatro sesiones adicionales de refuerzo cada tres meses durante un año adicional, las cuales fueron administradas por psicoterapeutas entrenados y supervisados mediante visualización aleatoria de entrevistas video-filmadas.

\section{Medición de resultados principales}

Se tomó como resultado principal el tiempo transcurrido a la recurrencia de un nuevo episodio depresivo (según criterios DSM-IV) medida en cinco intervalos durante 24 meses; y como resultados secundarios parámetros como cantidad de días libres de síntomas depresivos, síntomas depresivos residuales, comorbilidades médicas y psiquiátricas, calidad de vida y costo efectividad.

Los evaluadores desconocían el tratamiento recibido por los pacientes.

\section{Resultados principales}

El tiempo a la recurrencia de la depresión no difirió entre los tratamientos a lo largo de los 24 meses: 94/212 pacientes (44\%) en el grupo intervención, comparado con 100/212 (47\%) en el grupo control; Hazard ratio 0,89 (IC95\% 0,67 a 1,18). Los resultados secundarios tampoco mostraron diferencias entre grupos.

\section{Conclusiones}

No hay evidencias de que la TCBCP sea superior a la medicación antidepresiva de mantenimiento.

Ambos tratamientos se asociaron con resultados positivos en términos de disminución de recurrencias, síntomas residuales de la depresión y calidad de vida.

Fuente de financiamiento: National Institute for Health Research y Health Technology Assesment Programme.

\section{Comentario}

Los individuos con depresión recurrente (tres o más episodios) tienen un riesgo incrementado de sufrir nuevos episodios depresivos, razón por la cual se suele recomendar el uso medicación antidepresiva de mantenimiento durante al menos dos años luego de la última remisión sintomática'.

Uno de los grandes problemas en estos pacientes es el abandono del tratamiento, el cual ocurre por distintas razones que van desde los efectos adversos o la visión negativa de algunos pacientes respecto de tomar medicación psiquiátrica.

La TCBCP es un tratamiento desarrollado para enseñar habilidades cognitivas a personas con depresión con el objetivo de evitar la recurrencia. En la última década se ha observado un aumento considerable de la publicación de experiencias de tratamiento que utilizan el modelo de conciencia plena como estrategia terapéutica no solo para el tratamiento de la depresión recurrente ${ }^{2}$. La TCBCP inicialmente mostró resultados muy alentadores tanto al ser comparada con la ausencia de tratamiento como en comparación con un tratamiento farma- cológico antidepresivo de mantenimiento estándar ${ }^{3}$, aunque este reciente estudio así como otros similares ${ }^{4}$ sugieren tomar con cautela el optimismo inicial. Este ensayo clínico nos da información científica que permite apoyar una toma de decisiones compartidas en función de las preferencias del paciente. Sin embargo hay que considerar que este estudio se ha realizado sobre un grupo particular de pacientes, aquellos que estaban en tratamiento antidepresivo y se encontraban dispuestos a discontinuar la medicación para probar un tratamiento psicosocial, por lo que a la hora de generalizar estos resultados también debemos ser prudentes.

\section{Conclusiones del comentador}

La TCBCP se ofrece como una alternativa válida de tratamiento frente a los antidepresivos, posibilitando una elección de tratamiento en función de las preferencias del paciente y aumentando con ello la adherencia al mismo.

Guido Pablo Korman [ Consejo Nacional de Investigaciones Científicas y Técnicas, Universidad de Buenos Aires, Facultad de Psicología. guidokorman@ conicet.gov.ar ]

Korman G. La terapia cognitiva basada en la conciencia plena no fue superior a los antidepresivos en la prevención de la depresión recurrente. Evid Act Pract Ambul. 2015;18(4):108. Oct-Dic. Comentado de: Kuyken W, y col. Effectiveness and cost-effectiveness of mindfulness-based cognitive therapy compared with maintenance antidepressant treatment in the prevention of depressive relapse or recurrence (PREVENT): a randomised controlled trial. Lancet. 2015;386(9988):63-73. PMID: 25907157.

\section{Referencias}

1. National Institute for Health and Care Excellence. Depression in adults: recognition and management (en línea). Manchester, Inglaterra: National Institute for Health and Care Excellence; 2009. Disponible en URL: http://www.nice.org.uk/guidance/cg90/chapter/1-recommendations\#continuation-and-relapse-prevention (último acceso diciembre 2015). 2. Korman GP, y col. El modelo de Terapia Cognitiva Basada en la Conciencia Plena (mindfulness). Rev Arg Cl. 2012;21(1):5-14.

3. Garay CJ, y col. Terapia Cognitiva basada en Atención Plena (mindfulness) y la "tercera ola" en Terapias Cognitivo-Conductuales. Vertex 2015;119(26):49-56. 4. Williams JM y Col. Mindfulness-based cognitive therapy for preventing relapse in recurrent depression: a randomized dismantling trial. J Consult Clin Psychol. 2014;82(2):275-86. 